\title{
Jugend und transnationale Mobilität
}

\begin{abstract}
Eine einführende Betrachtung
Jugend und Mobilität gehören irgendwie zusammen. Dies ist eine Allerweltweisheit. Dennoch bedeutet dieser Zusammenhang für junge Menschen häufig sehr Unterschiedliches. Vor allen Dingen sind die sozialen Rechte und die Lebenslagen, durch die Mobilität im Jugendalter reguliert wird und in denen sie stattfindet, sozial ungleich ausgestaltet. Was für einige junge Menschen selbstverständlich erscheint, ist für andere gar nicht erst möglich. Diese Unterschiede spiegeln sich auch wieder, wenn die unterschiedlichen Formen transnationaler Mobilität im Jugendalter betrachtet werden.
\end{abstract}

$\mathrm{D}$ ie Mobilität von jungen Menschen macht nicht an den Nationalgrenzen halt, sondern geht weit darüber hinaus - ob im Rahmen von $\mathrm{Fa}$ milienbeziehungen, auf der Flucht, im Kontext von Arbeitsprozessen oder auch beim Reisen, im Studium, bei der Jobsuche usw.; ob freiwillig oder erzwungen. Unter transnationaler Mobilität ist ebenfalls gefasst, dass es nicht lediglich von A nach B geht, sondern dass die Überschreitungen von Nationalgrenzen vielfältig sind, dass es Pendelbewegungen zwischen A und B gibt und darüber auch neue transnationale Beziehungen und Räume entstehen. Der Beitrag von Agnetha Bartels in unserem Schwerpunkt beschäftigt sich mit einer spezifischen Form der transnationalen Mobilität, dem couchsurfing.

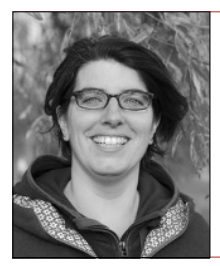

Katharina Mangold

Hildesheim, Deutschland

*1979; Dr., Diplom-Pädagogin, wissenschaftliche Mitarbeiterin am Institut für Sozial- und Organisationspädagogik der Universität Hildesheim.

katharina.mangold@uni-hildesheim.de

Wolfgang Schröer
Hildesheim, Deutschland
*1967; Dr., Professor an der Universität Hildesheim.
$\begin{aligned} & \text { Arbeitsschwerpunkte: Kinder- und Jugendhilfe, Transnationale } \\ & \text { Soziale Arbeit. } \\ & \text { schroeer@uni-hildesheim.de }\end{aligned}$

Zusammenfassung Einleitung in den Schwerpunkt "Jugend und transnationale Mobilität“, die die Hauptaspekte der Beiträge kurz skizziert.

Schlüsselwörter Jugendliche, (transnationale) Mobilität, Vernetzung, Ungleichheit
Was haben Jugend und transnationale Mobilität miteinander zu tun?

Wer den Zusammenhang von Jugend und (transnationaler) Mobilität geschichtlich etwas verfolgt, wird schnell feststellen, dass die damit verbundenen sozialen Phänomene in einem engen Zusammenhang stehen. Zumindest in der Geschichte moderner Industrie- und heutiger Wissensgesellschaften sind Jugend und Mobilität geradezu zwei Seiten einer Medaille.

Jugend ist auch eine Geschichte erzwungener Mobilität vom Land in die Stadt, aber auch über die Grenzen der entstehenden Nationalstaaten hinweg - hinein in die entstehenden Industriezentren und - heute - in die globalen Metropolen sowie auf der Flucht vor dem Hintergrund von Kriegen, Kinderrechtsverletzungen, Naturkatastrophen und sozialen Notlagen.

Gleichzeitig wird in modernen Gesellschaften Mobilität von jungen Menschen geradezu erwartet. Und es werden mitunter transnationale Mobilitätserfahrungen nahezu als Selbstverständlichkeit angesehen. Ob als Au-pair, als Globetrotter_innen, im Internationalen Freiwilligendienst, im Schüler_innenaustausch oder im Auslandsstudium - junge Menschen sammeln transnationale Erfahrungen und sind transnational vernetzt. Der Beitrag von Andreas Herz fragt nach dem Zusammenhang von transnationaler Mobilität und transnationaler Vernetzung. Im Beitrag von Katharina Mangold geht es dann um Incoming-Freiwillige und deren sozialen Netzwerke.

\section{Wem wird welche transnationale Erfahrung ermöglicht?}

So ist zu fragen, von wem welche Mobilität erwartet und wem welche transnationale Erfahrung sozial ermöglicht wird und welche Mobilität zu Stigmatisie- 


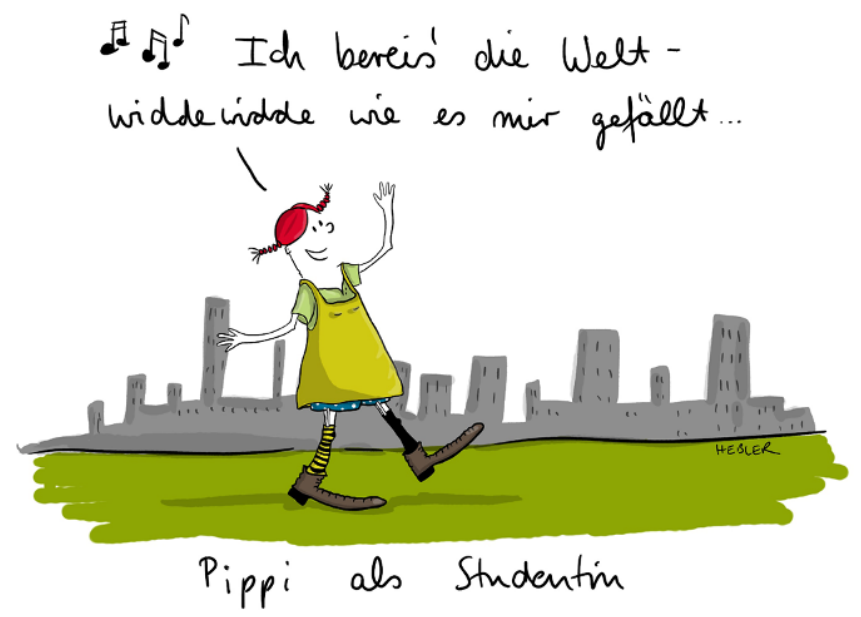

(c) Ruth Hebler

rungen und Ausgrenzung führt. Nicht nur im Rahmen internationaler Freiwilligendienste, wo empirisch nachgezeichnet werden kann, dass insbesondere bildungsorientierte junge Menschen sich an den Programmen beteiligen, muss die Frage gestellt werden, wie Angebote strukturiert sind und welche jungen Menschen sie adressieren. Der Beitrag von Alina Kierek und Moritz Decker macht genau dies zum Thema und stellt Projekte vor, in denen junge Menschen angesprochen werden, die bislang wenig erreicht wurden.

\section{Welche Auslands- bzw. Mobilitätserfahrung wird wie anerkannt?}

Während Mobilität - in der sozialwissenschaftlichen Diskussion - als Rahmenkonzept für unterschiedliche Formen lokaler Veränderungen genutzt werden kann und somit Veränderungen von Bevölkerungsstrukturen in den Blick genommen werden können, scheint die soziale und politische Strukturierung von Mobilität im Jugendalter eine zentrale Herausforderung der Jugendpolitik und -forschung. Denn nicht nur durch den politischen Diskurs werden die Mobilitäten junger Menschen sozial sehr unterschiedlich bewertet und mitgestaltet, sondern auch in der sozialwissenschaftlichen Forschungslandschaft.

Jugendmobilität erscheint hier mitunter als Errungenschaft und Motor der Veränderung. Sie wird insbesondere mit ökonomischen Aufstiegsperspektiven oder Wohlstand sowie kultureller Bildung und zivilgesellschaftlicher Entwicklung verbunden. Hierbei wird vor allem die Perspektive der verantwortungsvollen Bürger_in postuliert und in der transnationalen Mobilität die Entwicklung von Weltoffenheit und interkultureller Kompetenzen angenommen. Migration und Flucht werden z. B. hingegen eher negativ stigmatisiert und häufig mit sozialen Belastungen und Exklusion verknüpft.
Hier stehen also nicht die vorhandenen transnationalen Erfahrungen und Kompetenzen einer transnationalen Lebensgeschichte im Fokus. Der Beitrag von Kathrin Klein-Zimmer beschäftigt sich mit den unterschiedlichen Zuschreibungen zu Migration und Mobilität und thematisiert die damit verbundene Ungleichheit.

In diesem Zusammenhang hat Stephan Sting darauf hingewiesen, dass diese Entwicklung zu einer gespaltenen Mobilitätspolitik in Europa führt. Gespalten sei die Politik, weil sie sozial ungleiche Mobilitätschancen forciere. Mobilität sei dabei ein Privileg von europäischen jungen Bürger_innen, von denen ein ökonomischer und zivilgesellschaftlicher Nutzen erwartet wird; Abschottung müssen dagegen diejenigen erleben, die nicht unmittelbar als Ressource erkannt werden, die dann häufig als Migrant_innen bezeichnet werden (Schröer und Sting 2003).

Funding. Open Access funding provided by Projekt DEAL.

Open Access. Dieser Artikel wird unter der Creative Commons Namensnennung 4.0 International Lizenz veröffentlicht, welche die Nutzung, Vervielfältigung, Bearbeitung, Verbreitung und Wiedergabe in jeglichem Medium und Format erlaubt, sofern Sie den/die ursprünglichen Autor(en) und die Quelle ordnungsgemäß nennen, einen Link zur Creative Commons Lizenz beifügen und angeben, ob Änderungen vorgenommen wurden.

Die in diesem Artikel enthaltenen Bilder und sonstiges Drittmaterial unterliegen ebenfalls der genannten Creative Commons Lizenz, sofern sich aus der Abbildungslegende nichts anderes ergibt. Sofern das betreffende Material nicht unter der genannten Creative Commons Lizenz steht und die betreffende Handlung nicht nach gesetzlichen Vorschriften erlaubt ist, ist für die oben aufgeführten Weiterverwendungen des Materials die Einwilligung des jeweiligen Rechteinhabers einzuholen.

Weitere Details zur Lizenz entnehmen Sie bitte der Lizenzinformation auf http://creativecommons.org/licenses/by/4.0/deed.de

\section{Literatur}

Schröer, W., \& Sting, S. (Hrsg.). (2003). Gespaltene Migration. Wiesbaden: VS. 